\title{
Reflexiones clínicas sobre la grabación de entrevistas de atención psicológica ¿Qué podemos pensar sobre la situación en que el paciente pide grabar su entrevista psicológica?
}

\section{Clinical reflections on the recording of psychological care interviews. What can we think about the situation where patients ask that their psychological interview is recorded?}

\author{
Alfred Tejedo García \\ Universitat Autonoma de Barcelona, España
}

\begin{abstract}
Resumen: Este trabajo pretende reflexionar sobre la posibilidad de la demanda de grabación de intervenciones psicológicas, a petición del paciente. Realizamos consideraciones clínicas atendiendo a distintos supuestos: el paciente adulto, el paciente infantil y extrapolamos esas reflexiones a contextos de atención grupal (parejas, familias, grupos). La práctica de la psicología clínica requiere el ejercicio de la responsabilidad prudente implicando al paciente en el proceso de reflexión. La demanda de grabación creemos puede girar en torno a la impresión subjetiva de algo que falta de la cual hay que hacer consciente al paciente. Por otro lado, para sostener el pacto de cuidados implícito en toda atención clínica es necesaria la confianza entre el paciente y el clínico. En conclusión, se trata de ayudar al paciente que solicita ayuda psicológica a incrementar su capacidad de reflexión, a potenciar su autonomía y su esperanza de poder ver las cosas de otra manera antes que ceder de manera acrítica a los riesgos que comporta una grabación.
\end{abstract}

\footnotetext{
Para citar este artículo: Tejedo García, A. (2021). Reflexiones clínicas sobre la grabación de entrevistas de atención psicológica ¿Qué podemos pensar sobre la situación en que el paciente pide grabar su entrevista psicológica? Revista Clínica Contemporánea, 12(1), Artículo e2. https://doi. org/10.5093/cc2021a2
}

Alfredo Tejedo García es Psicólogo Clínico en el Centre de Salut Mental d'Adults Badalona 2/ Unitat d'Atenció Psicològica-Servei Assitencial de Salut de la Universitat Autonoma de Barcelona, España.

La correspondencia sobre este artículo debe enviarse al autor a los e-mail: alfred.tejedo@uab.cat;alfred.tejedo@gmail.com

(cc) BY-NC-ND Este es un artículo Open Access bajo la licencia CC BY-NC-ND. 
Palabras clave: grabación, intervención psicológica, ética, pacto de cuidados, código deontológico

\begin{abstract}
This work is intended to reflect on the possibility that patients request that psychological interventions are recorded. Clinical considerations are made based on different assumptions, including adult or pediatric patients, and those reflections are extrapolated to group care settings (couples, families, groups). The practice of clinical psychology requires the exercise of prudent responsibility involving the patient in the reflection process. We think that the demand for recording may revolve around the subjective impression that something is lacking, of which the patient must be made aware. On the other hand, to uphold the covenant of care implicit in all clinical care, trust between the patient and the clinician is required. In conclusion, patients who request psychological help should be helped to increase their ability to reflect, to enhance their autonomy and their hope to be able to see things differently, rather than uncritically yielding to the risks involved in a recording.

Keywords: recording, psychological intervention, ethics, covenant of care, deontological code
\end{abstract}

El móvil que inspiró la redacción de este trabajo fue la discusión que se generó en las sesiones del comité de bioética del cual soy miembro. Un profesional sanitario planteaba la cuestión de si un paciente podía grabar la intervención del profesional. En el seno del comité de ética asistencial hospitalaria hay profesionales de la práctica médica, de enfermería, juristas, farmacéuticos y psicólogos clínicos. Mi adscripción, como psicólogo clínico, al ámbito de la salud mental, me hacía situarme en una perspectiva diferenciada respecto de la simple concesión de grabar una intervención del profesional de enfermería o médico, en relación con la atención a una situación de cura de la enfermedad - del cuerpo, no de la mente- para uso del propio paciente o para el de algún familiar ${ }^{1}$.

Hoy en día se sostiene que el deseo del paciente de grabar su atención clínica tendría soporte legal, incluso aunque el clínico no se mostrara de acuerdo (Elwyn y Buckman, 2015; Rieger et al., 2018; Rimmer, 2019). Creemos que es una función de las Ciencias Sociales y, en particular, de la Psicología, el hacer ejercicio de la responsabilidad ética e ir detrás, no sólo del ethos burocrático, que busca dar cobertura al mínimo legal sino del ethos profesional, que busca la excelencia y la calidad de la atención (Sánchez, 2008; Sánchez, Lahitte y Ortiz, 2015). Así no nos resulta suficiente quedarnos con la perspectiva de lo que supuestamente es legal. Creemos que es necesario dar respuesta a este interrogante desde la perspectiva de cuando menos dos de los principios que animan la bioética, el de beneficiencia y el de autonomía, tratando de buscar el máximo beneficio con el mínimo daño para el paciente, desde el respeto por su autonomía (Yepes y Ocampo, 2018).

Estamos inmersos en una sociedad en que las tecnologías de la información y de la comunicación modulan la cotidianidad de las relaciones sociales, culturales y económicas superando todo tipo de barreras de espacio y tiempo. La sociedad de la información permite el acceso a infinitud de datos y a su difusión prácticamente ilimitada; el conocimiento se nutre de la información en bruto para interpretar los datos de acuerdo con los contextos que les pueden dar sentido. En nuestro caso, como profesionales de la salud mental, nos toca colaborar con el paciente en la asignación del sentido de su sufrimiento contextualizado al nivel de su realidad, co-construyéndolo con él, yendo más allá de la mera acumulación de información.

La irrupción de las nuevas tecnologías en nuestra vida habitual facilita ese planteamiento del paciente que muestra su deseo de grabar lo que sucede en la consulta, o una parte, o su totalidad. Hoy todo el mundo puede entrar en la consulta del profesional de la salud mental con el móvil, un smartphone o una tableta, instrumentos que permiten grabar, videograbar o audiograbar lo que está sucediendo en el momento casi sin que uno se dé cuenta. No obstante, se impone la reflexión a propósito de la idoneidad de la concesión de realizar ese interés por la grabación de la consulta; reflexión que nos incumbe personalmente. Podemos decir - con Ricoeur- que cuando recibimos la palabra del otro, se crea una situación nueva de la que no somos los dueños, dado que esa palabra ha producido un descentramiento (Sánchez, 2008). Este descentramiento que produce la palabra del otro tiene que ser atendido desde la reflexión prudente. La reflexión cuidadosa es una 
actitud requerida particularmente en situaciones novedosas para el psicólogo (Colegio Oficial de Psicólogos de España, 2004, pp. 126-129).

\section{La grabación de la atención clínica con fines de investigación}

La práctica de la grabación con fines de investigación se viene practicando desde los profesionales de la salud desde hace largos años y está avalada por la bibliografía prácticamente desde la década de los 50 del siglo XX (Redlich, Dollard, Newman, 1950; Lamb y Mahl, 1956; Silk, 1972; Marks, Montgomery y Davis, 1975; Fog-Petersen, May y Arnfred, 2015). La comunidad científica lleva años practicando, pues, la grabación de la actividad clínica con fines de investigación, a petición del clínico, y con consentimiento del paciente. Está claro que, sin consentimiento del paciente, esa grabación no cumpliría con los requisitos éticos para su realización. Con todo, es motivo de cuestionamiento la influencia que la grabación tiene sobre clínicos y pacientes; en particular se constatan los efectos adversos de la grabación en aquellos pacientes que tienen malestar psicológico (Gelso, 1973; Howe, 1997). Ciertamente, la grabación de la atención clínica también permite posibilidades de aprendizaje, para los clínicos, en situaciones de docencia. Con todo, esta práctica no está sostenida por todos los clínicos, es decir, no todos se muestran partidarios de ese proceder; otra cosa es que sí se obtengan beneficios, ¿solamente para la investigación?, pero, y ¿para el paciente? ¿Puede la grabación de la clínica beneficiar a los pacientes?

\section{¿Grabación de la atención clínica en beneficio del paciente?}

En la literatura científica los primeros trabajos que refieren los beneficios de la provisión de grabaciones de las consultas médicas a los pacientes se remontan a 1977 (Butt, 1977). Los beneficios de la grabación de la consulta clínica están referidos a áreas de la medicina y a situaciones muy concretas: en los ámbitos de la oncología, la pediatría de niños gravemente enfermos, la cirugía cardíaca y ortopédica, la atención prenatal y la atención primaria. Su utilidad se ha constatado en situaciones de diagnóstico de enfermedad grave en que la recepción del diagnóstico compromete en mucho la emocionalidad de la persona en detrimento del procesamiento cognitivo de la información; igualmente, en situaciones socioeconómicas muy limitadas, en personas muy mayores con cierto deterioro cognitivo o en pacientes con ciertas discapacidades, por ejemplo, la sordera (Tsulukidze et al., 2014). Ciertamente que hay una tendencia cada vez mayor, fruto de la era digital en la que vivimos, que hace más frecuente esta posibilidad, argüida por la complejidad de la información médica - una más que otra - , por su lenguaje abstruso y por la necesidad, pues, de clarificación y comprensión por parte del abrumado paciente (Rimmer, 2019). Y habría que preguntarse ¿no habría otra alternativa más empática con la situación del paciente antes que la de proveerle con una copia de la consulta?

La pretensión del paciente de grabar la consulta es un planteamiento que puede tener distintas respuestas, tanto porque puede haber partidarios y detractores de la concesión de grabación, como por la diversidad de lo que puede ser grabado. Los partidarios consideran que la grabación aporta indudables beneficios al paciente, en términos de seguridad y de calidad, por cuanto promueven el compromiso del paciente y, además, sugieren que el médico no tiene nada que esconder (Tsulukidze et al., 2014; Elwyn y Buckman, 2015; Rieger et al., 2018; Rimmer, 2019). Pero entre los detractores se considera que la grabación, como toda performance, supone una interferencia en la relación médico-paciente, que altera la funcionalidad de la consulta por cuanto los protagonistas de la experiencia se hacen conscientes de que tienen que actuar con la consecuencia de que se ve afectada la calidad de la atención (Elwyn y Buckman, 2015; Rieger et al., 2018); ciertamente, el estudio de Pringle y Stewart-Evans (1990), con unos clínicos que saben que están siendo grabados y otros no, lo desmiente, por cuanto no observa diferencias en el comportameinto de unos y otros. Los detractores consideran que sería más aconsejable buscar alternativas que puedan satisfacer la demanda de la grabación, por ejemplo, proveer al paciente de anotaciones escritas de acuerdo con la necesidad de la grabación, que algunos estudios refieren en el mismo nivel de beneficio para el paciente (Tsulukidze et al., 2014). 
Todos los trabajos consultados hacen referencia, no obstante, al balance necesario entre riesgos y beneficios de proveer al paciente de material de grabación, entre otras razones, por la necesaria posibilidad del escenario legal en caso de litigios, por eso se sugiere asesoramiento legal previo (Tsulukidze et al., 2014; Rieger et al., 2018). Con todo, en el caso de optar por la concesión a la grabación se aconseja que se queden copias del material ambas partes (Tsulukidze et al., 2014; Elwyn y Buckman, 2015; Rieger et al., 2018). Está claro que la sola posibilidad de la grabación remite al posible escenario del litigio.

Se trata, pues, de una cuestión polémica en la que caben posicionamientos diferentes en función de los presupuestos que puedan asumir de manera plural tanto los profesionales como los pacientes. Vemos dos escenarios posibles a partir del planteamiento de la demanda de la grabación a iniciativa del paciente: a) existe un acuerdo entre el profesional y el paciente respecto de la posibilidad de grabación de la atención clínica; b) no hay acuerdo, el paciente desea la grabación, pero el profesional no la consiente. Ahora hay que ver la motivación que mueve al paciente a realizar una petición así. Para ello atenderemos primero al posible alcance de la petición, es decir, qué se pretendería grabar.

El abanico de posibilidades para realizar la grabación es amplio. La motivación — consciente - que sostiene la demanda del paciente, quizás también. Si nos ceñimos a los trabajos publicados sobre las grabaciones de consulta - todos pertenecientes al ámbito de la medicina ${ }^{2}$ - está claro que el beneficio para el paciente gira en torno a la posibilidad de procesar mejor la información recibida en la consulta, precisamente, por la revisión de la misma. Esta revisión ocurriría en el hogar del paciente, en solitario, sin el profesional, aunque, en un porcentaje alto, entre el $60 \%$ y el $69 \%$, compartiendo la grabación con familiares, amigos e, incluso, otros médicos (Tsulukidze et al., 2014; Rieger et al., 2018). Curiosamente, en el estudio de Rieger se observa que la necesidad de compartir con otros (familiares, amigos y otros médicos) cuando se trata de la carta resumen explicativa de la consulta, el porcentaje baja al 50\% (Rieger et al., 2018). También se constata que la audición y visión de la grabación no está exenta de reacciones de distrés y de ansiedad en los propios pacientes, entre el $3 \%$ y el 26’8\% (Rieger et al., 2018).

\section{Objetivo de este trabajo}

Las reflexiones de este trabajo van a focalizarse sobre la intención de grabar, videograbar o audiograbar, entrevistas de atención psicológica o psicoterapéutica, por parte de la persona del paciente. Nuestras consideraciones estarían vinculadas a nuestra mentalidad acorde con la escucha y con la comprensión psicoanalíticas.

\section{Especificidad de la atención psicológica}

El profesional psicólogo clínico, para desempeñar su actuación profesional, ha de estar no solamente capacitado en virtud de unos estándares de formación clínica sino que, además, ha de estar concernido por la preocupación por el otro, por su cuidado y, en consecuencia, por los valores éticos que inspiran su Código Deontológico, hasta tal punto que le obliga, en su artículo 24, "a rechazar la prestación de servicios psicológicos cuando hay certeza de que puedan ser mal utilizados" (Consejo General de Colegios Oficiales de Psicólogos, s. f.; Colegio Oficial de Psicólogos de España, 2004, pp. 113-117). Igualmente, en el contexto de la práctica de la telepsicología, es decir, de la prestación de atención psicológica usando, precisamente, las nuevas tecnologías, las buenas prácticas para el psicólogo le obligan a velar por la seguridad y la privacidad de la información; el psicólogo "debe velar porque el cliente respete las mismas medidas de seguridad ya que de nada sirve que las asuma él pero no haga lo mismo su cliente" (Colegio Oficial de Psicólogos de Madrid, 2018). Al cliente se le advierte de la necesidad de la confidencialidad, que es la base de la alianza de trabajo, y, particularmente, se le hace firmar el consentimiento informado que exige la confidencialidad de la información intercambiada (Colegio Oficial de Psicólogos de Madrid, 2017). 


\section{Supuestos de la demanda}

Consideramos los supuestos que llevan a la demanda de querer grabar la sesión, por parte del paciente. Primero hay que diferenciar si el paciente es un adulto, o un niño que está en relación de dependencia de sus cuidadores. En segundo lugar, habría que diferenciar si se trata de la situación de la primera consulta o si se trata de una situación de encuadre de tratamiento psicológico.

\section{Caso de un paciente adulto}

El planteamiento de la grabación - que hasta la fecha no hemos vivido en primera persona, como clínicospuede significar revisar la sesión, a posteriori de la visita psicológica, para poder incorporar con más detenimiento los aspectos que se han tratado en tiempo real con el fin, por ejemplo, de mejorar el rendimiento de la atención clínica, de manera especial, en los encuadres en que se supone que hay una continuidad de visitas, de manera que se considerase que la optimización de lo trabajado en una sesión podría favorecer el abordaje de lo que se va a tratar en la siguiente.

También podría tratarse de revisar la sesión para poder compartir con terceras personas el contenido de los aspectos trabajados en la intimidad del tiempo real de la sesión clínico-paciente. Estas posibles terceras personas, podrían ser allegadas al sujeto paciente, por ejemplo, la pareja, algún familiar o amistades cercanos, incluso, podría tratarse de otros profesionales que podrían tener una cierta ascendencia en el sujeto, de la misma profesión psicológica o no.

O se podría querer revisar la sesión para poder usar su contenido, sin restricción, incluso con efectos de divulgación en ámbitos públicos, más allá del entorno próximo al individuo, por ejemplo, a través de los diversos medios de redes sociales.

\section{Caso de un paciente infantil}

El caso de un paciente infantil tiene la peculiaridad de que no es el niño quien decide asistir a una consulta sino que, habitualmente, son sus cuidadores quienes lo hacen. En este caso habría que diferenciar si la voluntad de grabación es estrictamente del adulto con ascendencia sobre el niño, si esa voluntad de grabación es compartida por los ascendientes adultos del niño, por ejemplo, en la mayoría de los casos, sus padres, o si no lo es, si solamente lo desea uno y no el otro y, sobre todo, cual es el deseo del niño al respecto, si también desea que se grabe su sesión o no.

Podríamos pensar en los mismos supuestos que en el caso del paciente adulto, que puede decidir y desear por sí mismo, pero con matices diferenciales, es decir, querer grabar la sesión infantil: si se trata de un uso exclusivamente del niño, o de sus padres, o de terceros, o de la posible publicación de la misma.

\section{Otros casos}

Por último podríamos pensar en hacer una extrapolación de nuestras consideraciones a la situación de consultas de pareja, de familia y de grupo.

\section{Consideraciones respecto de la motivación de la consulta del adulto}

La ubicación de la demanda en su contexto va a tener resonancias diferentes, necesariamente. 


\section{La motivación de la demanda de grabación en el encuadre de las primeras visitas}

En este contexto, la motivación que podría ser más próxima a la consciencia del paciente podría ser el querer apreciar con más detalle la realización de la visita o visitas realizadas para obtener algún beneficio.

En primer lugar, en la visualización de esa copia de la realización de la consulta o consultas iniciales, se supone que una parte indispensable de la experiencia, es decir, el clínico, no estaría presente, especialmente si esa grabación se diseña para uso del paciente fuera de los límites donde la asistencia clínica ha tenido lugar. Siendo esto así, esa visualización, necesariamente, estaría excluyendo a una parte integrante de la experiencia clínica, el psicólogo, coprotagonista con el paciente.

Desde la perspectiva del paciente se puede argumentar que la revisión de la consulta hace referencia a cuestiones que tienen que ver con su intimidad y que esa condición le haría dueño, en cierto sentido, de la propiedad - si se pudiera hablar así - de esa consulta. Pero no es toda la verdad esa apreciación, puesto que hoy en día sabemos que lo que se juega en toda interacción humana tiene una bidireccionalidad que es co-creada por los protagonistas de esa interacción. Lo que sucede en una consulta psicológica es un sistema complejo que no puede explicarse por las características de sus partes, paciente y psicólogo, sino que es un emergente nuevo.

Así, esa situación de consulta inicial no es cuestión solo que incumba a la intimidad del paciente, sino que incumbe, también, a la intimidad del clínico que la realiza. Por lo tanto, la visualización posterior de esa consulta, sin contar con la presencia del clínico que la ha llevado a cabo en colaboración con el paciente, estaría suponiendo una cierta exhibición de la intimidad de aquel sin su participación, aunque fuese a los ojos del paciente, exclusivamente, sin ningún tercero. ¿A quién espera contemplar y escuchar el paciente, a sí mismo o al clínico? Si el paciente quiere observarse a sí mismo, ¿qué sentido tiene?; si quiere observar al clínico, también, ¿qué sentido tiene? Lo mismo para la observación de la interacción de ambos.

En el supuesto que a esa re-visión de la consulta se incorporara la persona del clínico, obviamente, las consideraciones tendrían otra valoración. Pese a ello, en ningún caso se entendería que esa supuesta circunstancia se realizara fuera de las instalaciones donde ha tenido lugar la atención psicológica y, menos aún, que eso ocurriera en el domicilio del paciente. Habría que evaluar el significado de esa visualización de la sesión realizada presencialmente por ambos protagonistas. Arborelius y Timpka (1990) refieren el estudio de un visionado de grabaciones realizadas en consultas de atención primaria en que se convocaba, una semana después de la misma, al comentario conjunto, médico y paciente, ante un tercero.

En segundo lugar, habría que pensar en los condicionantes internos del paciente que le llevan a identificar ese deseo de repasar la consulta efectuada in vivo fuera de los límites de la misma. Podríamos apreciar, como hipótesis, una cierta dosis de inseguridad respecto de la capacidad de conservar la experiencia realizada dentro de sí más allá de los límites de la consulta. ¿Por qué habría que recurrir a la revisión de la consulta fuera de ella? Si la pretensión de la demanda de grabación es la supuesta incorporación interna, para el paciente, de una ampliación de la comprensión de la consulta realizada, esta sola demanda desvela una expectativa de la mente del paciente que entendemos como una exageración que desvela esa inseguridad que venimos de nombrar. Es evidente que esta demanda y la expectativa interna que la sostiene puede ser una información altamente significativa para el desvelamiento de la comprensión de los factores que están determinando una parte de la consulta del paciente, muy probablemente, al menos por cuanto podrían estar informando de esa inseguridad o exigencia internas.

En tercer lugar, la realidad de tener un observador de la consulta puede modificarla, por cuanto se incorpora un observador externo, en forma de máquina de grabación, que informaría al paciente y al clínico de la existencia de ese tercero externo a la díada paciente-clínico. Esta simple información ha de ser codificada por ambas mentes, a no ser que se haga una disociación entre sana y patológica ¿Por qué? La cámara de grabación, en sus múltiples formatos, informa de algo muy simple, algo así como que su sola presencia evoca la realidad de que este suceder interno de la consulta podrá ser reproducido con posterioridad, en condiciones esperables - ajustadas al origen de la demanda - bajo el control del paciente, exclusivamente.

Esto tiene, en nuestra opinión, dos tipos de consecuencias; por un lado, el paciente puede escindirse entre el estar plenamente presente en el aquí y ahora y el después, argumentándose, conscientemente o no, que tiene una segunda oportunidad para asegurarse respecto del suceder de la consulta in vivo. Por otro lado, para el 
clínico la presencia de ese artefacto técnico puede tener un efecto, cuando menos, distractor también y obstaculizar de su estar plenamente relajado y atento al momento presente con su paciente en el aquí y ahora, por cuanto una parte de su consciencia puede evadirse del presente tratando de dar cabida a la posibilidad de ese tercero observador del suceder íntimo entre él y su paciente. Salta a la vista el elemento de contrariedad que este objeto de grabación puede suponer para el clínico. Arborelius y Timpka (1990), en el trabajo referido, informan de cómo los médicos no se muestran neutrales ante la realidad de la grabación de las consultas, sino que les altera en su diligencia, en su hablar más exhaustivo, de manera particular, ante situaciones de exploración ginecológica; esta afectación de los clínicos es superior a la que muestran los pacientes que, además, perciben la afectación de los médicos (Coleman, 2000).

En cuarto lugar, puede ocurrir que el paciente no caiga en la cuenta y escinda su consciencia de la atención a estas consideraciones implícitas a la realización de la grabación; ciertamente, la pericia del clínico puede ayudarle a hacerse consciente de los peligros que supone ese artefacto. Pero la otra posibilidad es que el clínico no sea consciente, tampoco, de lo que está implícito en la aceptación de la grabación de la consulta, al menos, inicialmente, antes de aceptarla ¿Qué aspectos inconscientes podrían estar aludidos en esta posibilidad, por parte del paciente y por parte del clínico?

El deseo de la grabación creemos que sugiere, entre otras, la posibilidad inconsciente del narcisismo. Verse a sí mismo puede comportar algo del goce narcisista, si supusiera la posibilidad de congratularse en la visualización, o en el otro polo, del goce masoquista, en el caso de que el paciente tuviese la tendencia de mirarse desde el vértice de la baja autoestima. Pero no solo esto, sino que verse a sí mismo en la consulta, necesariamente evoca la presencia del otro, del clínico, ante quien el paciente habla y, por lo tanto, de su observación, también. Una vez más, hay que entender cuál es el móvil consciente e inconsciente de esa expectativa de grabación.

Si el asentimiento partiera del clínico, bajo la aparente concesión de que revisar la consulta presencial ayudaría más que perjudicaría al paciente, o a la relación asistencial, este comportamiento clínico podría no contravenir la confianza inicial de la relación, si es que para el clínico no se le representa en su mente consciente un problema, una amenaza, pero sí podría suponer una concesión al encuadre clínico que, con toda probabilidad, tendría que ser abordado en entrevistas posteriores - si supusiéramos que eso pudiera suceder - y ¿cómo decir que no más tarde si no se ha dicho desde el principio? O ¿no se diría que no exponiéndose a una grabación ilimitada de sesiones por parte del paciente? ¿dónde estaría el límite? Así, podríamos estar ante la escena del paciente que sostiene la voluntad realizada, aprobada por el clínico, para grabar todas sus sesiones de psicoterapia para ser visionadas en solitario fuera de los límites de la consulta. Y el clínico sin mostrar ninguna preocupación por el control de ese material sensible en que se muestra a si mismo con su paciente. Ahora bien, si se hiciera un mal uso y él pudiera tener conocimiento, siempre quedaría el recurso a los tribunales. Se nos antoja que es un innecesario riesgo cuya solución estaría en la prudente evitación de esa grabación o grabaciones desde el primer momento, de común acuerdo.

\section{La pretensión de compartir la grabación con terceros}

Si supusiéramos que la visualización de la grabación convoca a otros sujetos además del paciente, a las consideraciones manifestadas habría que añadir otra que sería de naturaleza patológica, por cuanto creemos que ese compartir la intimidad de la propia consulta con el clínico con un tercero podría tener el matiz de conducta exhibicionista, mostraría la necesidad de exhibir su intimidad ante terceros, por próximos que pudieran ser, aunque esto fuera inconscientemente. Como clínicos tenemos la repetida experiencia de encontrarnos con pacientes que no permiten que haya observadores - familiares o no- en la consulta, ni acompañantes, mayoritariamente; solo un porcentaje discreto de pacientes, en situaciones de primera consulta pueden preferir ser acompañados por algún familiar cercano, muy a menudo, la pareja o los padres, aunque la experiencia clínica desvela que este proceder, sostener la compañía del otro en la consulta, no es conveniente para la inmensa mayorías de situaciones, excepción hecha de pacientes gravemente afectados mentalmente. Son una excepción a la regla. 
La falta del control de la información sensible que representa toda sesión con un profesional de salud mental, especialmente, si es psicólogo en función clínica, puede ser ignorada por el paciente, pero esa entrega aparentemente confiada de lo que a uno le pasa por dentro, mentalmente, al familiar de turno, puede volverse en contra del paciente, tarde o temprano. En muchas ocasiones hemos observado en la clínica una crítica respecto del problema de salud mental que presenta el familiar "paciente" por parte de muchos familiares, aparentemente seres queridos y que quieren a los suyos, a quien se puede hacer depositario de la locura, o de los problemas psicológicos que tiene, -“él sí, yo no, porque yo no voy al psicólogo”. Así, invitar a la contemplación de ciertos aspectos de la intimidad mental puede ser una frivolidad de la que nos hemos de sentir responsables, como clínicos experimentados conocedores de algunos aspectos de la humana naturaleza y, consecuentemente, proteger a nuestros pacientes, especialmente a los que pueden estar en una situación de mayor fragilidad, como lo pueden ser aquellos que acuden a la primera consulta con su psicólogo.

¿Y si se tratase de llevarle la grabación a otro profesional? A la consideración anterior habría que añadir la posibilidad de contravención del Código Deontológico, en su artículo 27, que refiere la posibilidad de negarse a simultanear la intervención con otro profesional (Consejo General de Colegios Oficiales de Psicólogos, s. f.).

\section{La motivación de la demanda en el curso del tratamiento}

En el caso de la motivación de la demanda en la situación de encuadre de tratamiento psicológico, tomaríamos en cuenta las consideraciones referidas a la situación de las entrevistas iniciales, con el añadido de ayudar a entender al paciente ese afán sobrevenido de grabación cuando ya se habría puesto en marcha el tratamiento, ¿por qué aparecería ahora?, ¿qué novedad estaría representando la situación del tratamiento? Por lo tanto, stricto sensu, esa demanda tendría que ser entendida desde la perspectiva de atender a su significado, tanto consciente, como inconsciente, si pudiera ser - cosa obligada en un encuadre de psicoterapia psicoanalítica-, paralelamente a atender a su contestación, desde nuestro punto de vista, de acuerdo no solo con lo innecesario de la misma sino de sus contraindicaciones. Así habría que contestar a la pregunta, negativamente, después de entender el sentido de la misma de acuerdo con la situación mental interna del paciente.

\section{La posible difusión pública de la grabación}

Concluyendo estas consideraciones hemos de añadir que la divulgación de las supuestas grabaciones de las consultas entre el paciente y el clínico en las distintas redes sociales sería una vulneración del derecho a la intimidad, al menos, de la persona del clínico y, por lo tanto, susceptible de ser delictiva. Razón de más para proteger al paciente y al clínico de lamentables escenarios que la falta de previsión en las situaciones iniciales de la consulta o la falta de reflexión ponderada de esta delicada circunstancia pudieran pasar por alto.

\section{Consideraciones respecto de la motivación de la consulta del niño}

A lo largo de los años de la infancia se va a dar un proceso a favor de la madurez y a medida que nos alejamos de la edad próxima al nacimiento se va a producir un acercamiento a la posibilidad de asunción de responsabilidades que, incluso, tendría el aval de la legislación. Se suele considerar, legalmente, la edad de 12 años como punto de corte de una cierta capacidad de autonomía y de decisión respecto, por ejemplo, de aspectos que tienen que ver con la elección de procedimientos en el ámbito de la salud de la persona del niño, no desvinculados del consentimiento de los padres o de quienes tuvieran su tutela.

En el ámbito de la salud mental, las primeras consultas realizadas para el niño puede que las pida este, excepcionalmente, y que transmita su deseo a sus padres - a mayor edad del menor, mayor probabilidad de que ocurrapero lo común es que sea llevado a consulta o por deseo de sus padres, o por indicación de terceros profesionales o, incluso, por mediación de terceros autorizados, como los profesionales del ámbito de la protección a los menores. 
Podríamos suponer que fuera el niño quien quisiera que se efectuara la grabación, en ese caso habría que hacerle consciente, en la medida de lo posible, de los condicionantes que están en juego en la realización de esa posibilidad, así como de sus riesgos, al nivel de sus capacidades, teniendo en cuenta las consideraciones que hemos referido pensando en el supuesto del paciente adulto.

Cuando fuesen los padres los que pidiesen la grabación, habría que proceder de la misma manera, añadiendo la conveniencia de incorporar a la reflexión al mismo niño, en función de su edad y de sus capacidades, dado que estaríamos ante una situación en que se juega la intimidad del niño, que sería quien estaría en el lugar del paciente. Por lo tanto, habría que devolver, de alguna manera, la conveniencia de confiar en el natural suceder de la exploración clínica inicial más que en el recurso a artefactos inapropiados y de cierto riesgo de acuerdo con las consideraciones señaladas pensando en la mente adulta del paciente adulto.

Podría suceder que uno de los padres no pudiese asistir a la realización de la consulta — a menudo es la figura del padre - y que hubiese mostrado el deseo de poder participar de alguna manera. Esta posibilidad podría suplirse, mejor, con la realización de otro recurso técnico como sería la videoconferencia en directo de acuerdo con los procedimientos apropiados para la práctica de la telepsicología, es decir, con las suficientes medidas de seguridad y de privacidad (Consejo General de la Psicología de España (2017).

Cuando los padres están separados la demanda de grabación debería ser entendida, igualmente, de acuerdo al caso concreto y ver cómo plantear la respuesta sin que se vulneren los derechos de nadie, de manera especial, del progenitor ausente.

Si se tratase de la voluntad de compartir con un tercero profesional, médico pediatra o de cualquier otra especialidad, o paraprofesional, el peligro sería lo que hemos argumentado como vulneración del derecho a la intimidad, en este caso, tanto del niño como del profesional clínico y, en alguna medida, de los mismos padres, si se tratase de la supuesta grabación en que ellos estuvieran presentes. En el caso de la voluntad de grabar la consulta con la expectativa de difundirla en las redes sociales, también habría que aplicar la misma argumentación presentada para la situación del paciente adulto, es decir, que contraviene el derecho a la intimidad y que sería susceptible de ser un acto delictivo.

\section{Extrapolación a la consulta de parejas, familias y grupos}

Los argumentos explicitados para ambas edades de pacientes, niños y adultos, en encuadres de atención psicológica individual también aplican a los encuadres de grupo, sean parejas, familias o grupos terapéuticos, tanto de pacientes adultos como de niños. La grabación de esas supuestas consultas o sesiones de psicoterapia, afectarían, no sólo a la individualidad de los que estarían en el lugar de los pacientes, niños o adultos, sino a la persona del profesional clínico. La grabación de imágenes, audios o videos, en situación de atención psicológica o psicoterapéutica son contenidos que directamente pertenecen a la esfera de datos personales y de salud, protegidos por la legislación vigente. Y ya hemos especificado que, aunque se tomase la supuesta decisión de grabación por parte de los que estarían en el lugar de los pacientes, en este caso, grupales, parejas o familias, habida cuenta de que el clínico es parte activa de la misma experiencia, su intimidad también estaría concernida y los riesgos que de esa práctica se derivarían los hemos explicitado ampliamente en los supuestos previos.

\section{Discusión}

a) Desde una perspectiva psicoanalítica entendemos que lo que mueve al sujeto en una dirección está determinado por algo que no se restringe a la mera motivación consciente del mismo. En psicoanálisis se entiende que hay una buena parte de la motivación humana que no es consciente, que es inconsciente. La conducta humana se encuentra animada por fuerzas motivacionales de procedencia endógena que Freud denominó pulsiones (Moore y Fine, 1997). A diferencia del instinto en los animales que les impele a búsqueda de la satisfacción, en el ser humano, no está garantizada esa satisfacción aunque, supuestamente, la pulsión la pretenda. La pulsión, que en Freud tiende a la búsqueda de la satisfacción 
en el contacto con su objeto, en Lacan, no puede ser satisfecha porque no apunta al objeto sino que gira perpetuamente en torno a él (Evans, 1997).

En la demanda del paciente de querer grabar su consulta con el clínico se explicita su deseo, así como se puede inferir lo que es la causa de él, lo que le mueve internamente a considerarlo. Lo que el sujeto plantea con su demanda de querer grabar, implícitamente, muestra su deseo de no querer perderse algo de la realidad, si el objetivo recae en un uso para sí mismo, sea para la visualización en solitario o compartida, o de copiar la realidad para su uso, supuestamente, ante terceros. Proyecta sobre la situación de la consulta una sensación de falta que lleva consigo. Así, está poniendo en palabras su configuración interna - por decirlo así - que le hace decir que le va a faltar algo de la realidad de la consulta que no va a poder llevarse con él - sin la grabación. A nuestro entender psicoanalítico, el clínico no debería pasar por alto esta observación, ni mucho menos reforzarla, ni coludir con ella, sino, en todo caso, ayudar a que la viera el paciente, que se hiciera consciente de ella y, por tanto, a tolerarla.

Supuestamente, concediéndole al paciente la grabación, el clínico estaría alimentando la negación del paciente de lo que es la configuración de la humana realidad, que no es otra que la necesaria alternancia de presencias y de ausencias. Además, conceder la grabación de la consulta es tanto como conceder que esa sensación de insuficiencia debería ser satisfecha fuera de los límites de la consulta, sin la colaboración del clínico. ¿Cómo aceptar que esa sensación interna debe ser atendida fuera de la consulta antes que dentro? ¿Por qué razón no podría destinarse más tiempo a ayudar a entender al paciente lo que parecería necesitar comprender dentro de los límites espaciotemporales de la consulta? Conceder la grabación ¿no sería favorecer la actitud de "piénselo usted, háblelo con los suyos y con la competencia si hace falta, o con su abogado, y ya me contará"?

¿No sería una función más propia del psicólogo clínico la de ayudar al paciente a ver qué es lo que sí puede entender, si es que es así, junto a lo que cree no poder entender y ver qué función representa lo uno y lo otro en su realidad psíquica? Piénsese, además, que, con toda probabilidad, la demanda de querer grabar la consulta, lógicamente, podría plantearse más al principio de la misma que no al final, cuando ya no habría prácticamente nada que grabar. Lo cual evidencia aún más la declaración de intenciones del paciente y, más todavía, su comunicación inconsciente implícita.

Ya en el principio de este trabajo hemos subrayado cómo la grabación de la consulta se viene realizando desde hace muchos años con fines de docencia y de investigación, a petición de los clínicos. Incluso, en algunos abordajes psicológicos - de corte sistémico - se utiliza este recurso que, sin duda, redunda en una mejor atención clínica. También es el caso cuando se trata de la formación en terapia basada en la mentalización, siempre a petición del clínico y con el consentimiento del paciente. Pero lo que se plantearía con la demanda del paciente que, en primer lugar, debería tener el consetimiento del clínico, apunta a un escenario muy distinto del que se plantea en los abordajes psicológicos mencionados, así como del control que el clínico tiene de ellos. Quien tendría el control de la información grabada sería el paciente. Aunque el paciente pudiera argumentar que sería para su mayor aprendizaje, se nos antoja que ese proceder, si no contara con la participación del clínico, sería un artefacto situado en paralelo de la asistencia clínica, consulta o tratamiento. Se impondría un cuestionamiento respecto de la eficacia de la intervención psicológica: ¿qué es lo que beneficia o cambia la situación del paciente? ¿la consulta en directo o la revisión de la misma en diferido?

Cabe la posibilidad de encontrarse con una demanda de grabación a los efectos de presentarla ante instancias de terceros (instituciones, tribunales, etc.) bajo la expectativa de obtener algun beneficio por los motivos que fueren. En ese caso, creemos que hay que aplicar la misma reflexión: ¿cuál es el sentido de esa expectativa?, ¿seguro que el beneficio que se busca sólo se puede satisfacer con la grabación?, ¿es el mejor medio o hay otros más indicados? También al inicio de este trabajo recogemos los aportes de la bibliografía en el ámbito sanitario que evidencian que la redacción de escritos es de gran utilidad y, de hecho, es la que se suele utilizar con ese objetivo (Tsulukidze et al., 2014).

b) La situación de la consulta remite a un escenario clínico peculiar que tiene connotaciones éticas, necesariamente. La consulta psicológica, en particular, y la atención sanitaria, en general, son un 
escenario en el que se produce el encuentro de dos personas, las dos concernidas por la ética. A este encuentro, que no es sino un espacio intersubjetivo, asisten cada uno, desde su autonomía y su vulnerabilidad y han de decidir conjuntamente (Sánchez, Lahitte y Ortiz, 2015). Estas dos personas, con roles netamente diferenciados, implícitamente, suscriben un pacto de cuidados que ha de estar basado en la confianza mutua, necesariamente; esto no obsta a que se pueda sentir la amenaza de la desconfianza, de la sospecha. Este pacto, lo suscribe el paciente haciendo depositario al clínico de un saber, o de un saber hacer, respecto de su sufrimiento. El reto del profesional será poder dar sentido a ese sufrimiento en el marco de una narrativa de vida del propio paciente, para lo cual es indispensable la palabra de este.

Por otro lado, esta situación de consulta está concernida por la deontología del profesional que, en tanto normativa, aspira a una cierta universalización de la relación interpersonal y que obliga al clínico a sujetarse a unas condiciones éticas del cuidado: privacidad, seguridad, confidencialidad. Al mismo tiempo, ambos protagonistas, clínico y paciente, se adhieren al derecho y al deber de saber la verdad, de manera especial, del paciente, a partir de la escucha de lo particular, del discurso del paciente; esta escucha es una escucha que no sabe más que a partir de lo que cuenta el paciente y que se construye, de descubrimiento en descubrimiento, aliada estrechamente con el sostenimiento del pacto de confianza (Domingo, 2007).

Necesita mención especial la situación de la grabación de la consulta cuando el sujeto concernido es el niño, que es un sujeto de derecho universal, protegido como tal sujeto por la legalidad vigente, que se encuentra entre la autonomía y la vulnerabilidad y, por tanto, con necesidad de defensa y cuidado especial. El niño, en función de su maduración, puede estar en condiciones de aprender a dialogar, a pensar críticamente, a estar conectado con su propio deseo, o no. Así, en la situación de la consulta clínica, el niño, en la medida de sus posibilidades, ha de ser invitado a tomar la palabra, de forma que no se le coloque en la situación de padecer su relación con el otro y que sea el otro el que decida sobre él desde las pretensiones del otro y no desde las de él (Sánchez, 2007).

Un interrogante: ¿Cómo podría realizarse una consulta psicológica entre dos personas cuando una de las dos pudiera no estar de acuerdo en algo sustancial como es la grabación de una consulta? ¿Cómo establecer un pacto de cuidados basado en la confianza a partir de un desacuerdo inicial tan radical, dijere lo que dijere la ley, supuestamente a favor del deseo del paciente?

Corresponde al clínico, y más si es psicoterapeuta, el favorecer que el paciente pueda poner en marcha sus recursos de capacitación desde el primer momento que se organiza una consulta en salud mental. Más que coludir con sus sentimientos de incapacitación, inseguridad, exigencia, confusión, como podría representar la idea de apoyarse en la muleta de la visualización de la consulta, se trata de devolverle al paciente la esperanza de que las cosas pueden ir de otra manera respecto de lo que cree en la situación en que se encuentra cuando va a consulta psicológica que, comúnmente, no es la situación de mayor autoconfianza. Así, desde el primer momento, se trata de ayudarle a realizar que puede asistir a su consulta sin ningún artefacto que confirme y amplifique, por ejemplo, - si fuera el caso - su temor de retener la información de lo que en ella sucede, potenciando, pues, su autonomía.

Por otro lado, esta actitud reflexiva respecto de la concesión de la grabación de la consulta favorece la diferenciación de lo que sería un espacio terapéutico, del orden de lo íntimo, de ayuda mental, respecto de lo que son otros espacios de su vida, menos íntimos, en los que sí que puede grabarse a sí mismo en compañía de otros sujetos - si así lo conceden - con toda la libertad que los límites de la legislación le permitiera.

La situación de la consulta en salud mental está emparentada con el ámbito de la intimidad de las personas que la realizan - de manera especial, del paciente - y las condiciones de su realización deben estar protegidas con las medidas oportunas para asegurar el derecho a la intimidad; por parte del profesional clínico deben estar protegidas, primeramente, por la suscripción del pacto de cuidados y, secundariamente, por el cumplimiento del secreto profesional acorde con el Código Deontológico ${ }^{3}$ (Consejo General de Colegios Oficiales de Psicólogos, s. f.). así como por las garantías de seguridad que la legislación contempla. Por parte del paciente, por la puesta en marcha de su autocuidado 
respecto del derecho a su intimidad y por el cuidado del otro, respecto al derecho a la intimidad de la persona que le atiende, ambas condiciones implícitas al pacto de cuidados. Concretamente, el artículo 39 del mencionado Código Deontológico sostiene que el psicólogo ha de ser escrupulosamente respetuoso con la intimidad del paciente.

Multitud de actos de los profesionales sanitarios de los distintos profesionales de la salud -médicos, enfermeros, trabajadores sociales, psicólogos, auxiliares, fisioterapeutas, terapeutas ocupacionales - desde los realizados en el consultorio de la atención primaria, o la atención especializada, o los realizados a nivel de hospitalización, incluidos los involucrados en las intervenciones quirúrgicas, no se realizan a la luz pública, sino en las condiciones de seguridad y privacidad que su práctica requiere ¿sólo por razones higiénicas sanitarias?

Cada cosa en su sitio. Supone cierta organización mental, más que menos, saber diferenciar los espacios en los que deben tener lugar unas y otras actividades del acontecer diario. En buena medida, una parte de nuestras vidas está sometida a las directrices que la sociedad de consumo ha conseguido inocularnos, así, por ejemplo, en los aconteceres más corrientes de nuestras vidas, desplazarse por la ciudad en transporte público, asistir a una comida a un restaurante, estar en una conferencia o acto docente, incluso en una reunión de trabajo, y tantas otras situaciones, se hacen prácticamente imposibles sin el uso del móvil, del smartphone o de la tableta - en ocasiones hasta el límite de la falta de respeto - o para consultar lo que se nos está sugiriendo desde el afuera de donde estamos, o para atender a una conversación con otra persona o grupo de whatshapp, o para inmortalizar ese suceder con una audiograbación o con una filmación.

Se puede llegar a la consulta en ese estado que la sociedad consumista nos inocula. Es frecuente encontrarse con pacientes que no desconectan el teléfono móvil antes de entrar a la consulta y, lamentablemente, suena el timbre de llamada, o el sonido de mensaje recibido en alguna de sus aplicaciones de mensajería, con lo que la interrupción de la consulta está garantizada, por breve que sea. No en vano cada vez son más visibles carteles que disuaden de su uso en los lugares sanitarios y de la administración pública. En este caso, habría que ayudar a nuestros confundidos pacientes a diferenciar que la consulta psicológica no es un ámbito cualquiera, sino que responde al ámbito de lo íntimo que no necesita de ningún artefacto externo en que apoyarse para su realización y que se puede obtener el beneficio al que se creería aspirar con la grabación, sin lugar a dudas, sin ella. La experiencia clínica lo acredita.

\section{Conclusiones}

El psicólogo está concernido por el ejercicio de la responsabilidad prudente que requiere reflexión, deliberación, de manera especial ante situaciones que se presentan como novedosas como es el caso de la supuesta demanda de grabación de la consulta. Así, el psicólogo debe ir más allá del planteamiento legalista que supondría la sumisión del clínico al deseo del paciente avalado por la ley, sin más. En el ámbito de lo médico se sugiere que en el caso de que se conceda la grabación, el clínico conserve una copia anticipando los posibles escenarios de litigio. Es, pues, una cuestión delicada.

El ejercicio de la reflexión cuidadosa, en el caso de posible grabación de la consulta, pasa por atender a la motivación del paciente, elucidando los propósitos tanto conscientes como inconscientes, y encontrar alternativas válidas que no signifiquen la simple concesión acrítica de la grabación; particularmente, desde la óptica psicoanalítica, se suele pretender primero entender el significado de la demanda del paciente antes que su ciega satisfacción.

El psicólogo debe atender a los riesgos que están implícitos en la grabación de la consulta y ofrecérselos a su paciente para que pueda elegir la opción menos arriesgada. Así, cabrá repasar el uso que se le querría dar a la supuesta grabación atendiendo a los peligros tanto de índole psicológica individual (falta de confianza en la capacidad de que la realidad de la consulta sea suficiente para el aprendizaje de lo que en ella sucede; posible exposición de la intimidad propia a ojos de terceros próximos al paciente o no), como de riesgos de actos supuestamente delictivos (ante la posibilidad de la difusión de lo grabado), como de los peligros de exclusión 
del clínico de la visualización de lo grabado - peor aún si es reiterada y sistemática - así como de los efectos contraproducentes para la atención completa en el aquí y ahora de la consulta, tanto para el paciente como para el clínico, ante la posibilidad de implementar un artefacto de observación de la misma, como en toda performance.

La particularidad de la consulta infantil añade complejidad a la demanda dado que el niño, su deseo, y su derecho a la intimidad, ha de tener cabida y ser respetado y no ha de ser subsumido en la necesidad o el deseo de sus tutores, quienes fueren.

Es notoria la diferencia entre la grabación de la consulta a efectos de formación e investigación, dado que, en esas circunstancias, el material grabado pasa a ser visualizado por un tercero desde el cual se hacen las apreciaciones sugestivas de una mejor atención clínica en beneficio, en última instancia, del paciente. La grabación para uso del paciente, o de los suyos, sin el clínico, no remitiría a esa instancia de terceridad desde la cual, se hace una comprensión - con el clínico, no sin él - que mejora la atención clínica. Como máximo, en el mejor de los casos, podría suceder una comprensión individual del paciente de algunas cuestiones de la consulta que al dejar al clínico fuera de ese ejercicio de comprensión podría tener consecuencias respecto de la apreciación de qué es lo que promueve el aquí y ahora respecto de la visualización en diferido.

Esperemos que en un futuro se puedan verificar, confirmando o denegando, las apreciaciones que consideramos de manera intuitiva basándonos en nuestra experiencia clínica hasta la fecha.

\section{Referencias}

Arborelius,E.y Timpka,T.(1990). In what way may videotapes be used to get significant information about the patient-physician relationship? Medical Teacher, 12(2), 197-208. https://doi.org/10.3109/01421599009006697

Butt, H. R. (1977). A method for better physician-patient communication. Annals of Internal Medicine, 86(4), 478-480. https://doi.org/10.7326/0003-4819-86-4-478

Colegio Oficial de Psicólogos de España (Eds.). (2004). Ética y Deontología para Psicólogos. http://www.cop. es/pdf/etica.pdf

Colegio Oficial de Psicólogos de Madrid (Eds.). (2017). Competencias del psicólogo para la presentación de servicios de TelePsicología. https://www.copmadrid.org/web/publicaciones/competencias-del-psicologo-para-la-prestacion-de-servicios-profesionales-de-telepsicologia

Colegio Oficial de Psicólogos de Madrid. (2018). Buenas prácticas de protección de datos en internet. https:// www.copmadrid.org/web/img db/publicaciones/buenas pract prot datos internet-pdf-5a9d4ca85de59.pdf Coleman, T. (2000). Using video-recorded consultations for research in primary care: advantages and limitations. Familiy practice, 17(5), 422-427. https://doi.org/10.1093/fampra/17.5.422

Col·legi de Metges de Barcelona. (16 de julio, 2019). Un pacient m'està enregistrant a la consulta. Ho pot fer?

[Entrada de blog] https://blogcomb.cat/2019/07/16/un-pacient-mesta-enregistrant-a-la-consulta-ho-pot-fer/

Consejo General de Colegios Oficiales de Psicólogos. (s. f.). Código deontológico del Psicólogo. Recuperado el 10 de noviembre de 2020 en http://www.cop.es/pdf/CodigoDeontologicodelPsicologo-vigente.pdf

Consejo General de la Psicología de España. (2017). Guía para la práctica de la Telepsicología. Autor. https:// doi.org/10.23923/cop.telepsicologia.2017

Domingo, T. (2007). Bioética y hermenéutica. La aportación de Paul Ricoeur a la bioética. Veritas, 2(17), 281312.

Elwyn, G. y Buckman, L. (2015). Should doctors encourage patients to record consultations? British Medical Journal, 350. Artículo g7645. https://doi.org/10.1136/bmj.g7645

Evans, D. (1997). Diccionario introductorio de psicoanálisis lacaniano. Paidós.

Fog-Petersen, C., May, My Arnfred, S. (2015). Exploring the Use of On-Site Tablet Video Recordings of Patient Interviews During Psychiatric Placement. Academic Psychiatry, 40(2), 392-393. https://doi.org/10.1007/ s40596-015-0469-8

Gelso, C. J. (1973). Effect of audiorecording and videorecording on client satisfaction and self-expression. Journal of Consulting and Clinical Psychology, 40(3), 455-461. https://doi.org/10.1037/h0034548 
Howe, A. (1997). Refusal of videorecording: what factors may influence patient consent? Family Practice, 14(3), 233-237. https://doi.org/10.1093/fampra/14.3.233

Lamb, R., y Mahl, G. F., (1956). Manifest reactions of patients and interviewers to the use of sound recording in the psychiatric interview. American Journal of Psychiatry, 112(9), 731-737. https://doi.org/10.1176/ ajp.112.9.731

Marks, S., Montgomery, J. S. y Davis, W. L. (1975). Videotape recording in counselling: 'What we know and where to find it'. Canadian Counsellor, 9(1), 31-44.

Moore, B. E. y Fine, B. D. (Eds.). (1997). Términos y conceptos psicoanalíticos. Biblioteca Nueva.

Pringle, M. y Stewart-Evans, C. (1990). Does awareness of being video recorded affect doctors' consultation behaviour? British Journal of General Practice, 40(340), 455-458.

Redlich, F. C., Dollard, J. y Newman, R. (1950). High fidelity recording of psychotherapeutic interviews. The American Journal of Psychiatry, 107(1), 42-48. https://doi.org/10.1176/ajp.107.1.42

Rieger, K. L., Hack, T. F., Beaver, K. y Schofield, P. (2018). Should Consultation Recording Use be a Practice Standard? A Systematic Review of the Effectiveness and Implementation of Consultation Recordings. Psycho Oncology, 27(4), 1121-1128.

Rimmer, A. (2019). My patient wants to record our appointment, what should I do? British Medical Journal, 364, Artículo 11101. https://doi.org/10.1136/bmj.11101

Sánchez, M. J. (2007). Ética e infancia: el niño como sujeto moral. Fundamentos en Humanidades, VIII(15), 179-192.

Sánchez, M. J. (2008). Ética y profesión: la responsabilidad en términos de prudencia responsable. El caso de la psicología. Fundamentos en Humanidades, IX(17), 145-161.

Sánchez, M. J., Lahitte, H. B. y Ortiz, V. (2015). Bioética y salud mental. Reflexiones sobre la afección y la responsabilidad en la relación profesional-paciente. Revista Latinoamericana de Bioética, 29(2), 96-107. https://doi.org/10.18359/rlbi.538

Silk, S. (1972). The use of videotape in brief joint marital therapy. American Journal of Psychotherapy, 26(3), 417-424. https://doi.org/10.1176/appi.psychotherapy.1972.26.3.417

Tsulukidze, M., Durand, M.-A., Barr, P. J., Mead, T. y Elwyn, G. (2014). Providing recording of clinical consultations to patients a highly valued but underutilized intervention: as coping review. Patient Education and Counseling, 95(3), 297-304. https://doi.org/10.1016/j.pec.2014.02.007

Yepes, C. E. y Ocampo, A. (2018). Comités de ética y salud mental. Revista Colombiana de Psiquiatría, 47(2), 129-136. https://doi.org/10.1016/j.rcp.2017.05.011

Artículo recibido: $18 / 05 / 2020$

Artículo aceptado: 13/01/2021 
Notas de autor

${ }^{1}$ El Col·legi Oficial de Metges de Barcelona (2019) tiene una página web en que aborda el tema de la demanda de grabación de la consulta médica en la que se plantean los límites de esa demanda.

${ }^{2}$ No hemos encontrado ningún trabajo referido al ámbito de la psicología.

${ }^{3}$ Particularmente en sus artículos 40, 46 y 49. Artículo 40: “Toda la información que el/la Psicólogo/a recoge en el ejercicio de su profesión, sea en manifestaciones verbales expresas de sus clientes, sea en datos psicotécnicos o en otras observaciones profesionales practicadas, está sujeta a un deber y a un derecho de secreto profesional, del que sólo podría ser eximido por el consentimiento expreso del cliente". Articulo 46: "Los registros escritos y electrónicos de datos psicológicos, entrevistas y resultados de pruebas, si son conservados durante cierto tiempo, lo serán bajo la responsabilidad personal del Psicólogo en condiciones de seguridad y secreto que impidan que personas ajenas puedan tener acceso a ellos". Artículo 49: "El fallecimiento del cliente, o su desaparición -en el caso de instituciones públicas o privadas- no libera al Psicólogo de las obligaciones del secreto profesional". 